\title{
Lubricin as a novel nanostructured protein coating to reduce fibroblast density
}

This article was published in the following Dove Press journal:

International Journal of Nanomedicine

25 June 2014

Number of times this article has been viewed

\author{
George Ejiofor Aninwene II' \\ Zifan Yang ${ }^{2}$ \\ Vishnu Ravi ${ }^{3}$ \\ Gregory D Jay 2,4 \\ Thomas J Webster ${ }^{1,5}$ \\ 'Department of Chemical Engineering \\ and Program in Bioengineering, \\ Northeastern University, Boston, \\ MA, USA; ${ }^{2}$ School of Engineering, \\ Brown University, Providence, RI, \\ USA $;{ }^{3}$ Albany Medical College, Albany, \\ NY, USA; ${ }^{4}$ Department of Emergency \\ Medicine, Brown University, School \\ of Medicine, Providence, RI, USA; \\ ${ }^{5}$ Center of Excellence for Advanced \\ Materials Research, King Abdulaziz \\ University, Jeddah, Saudi Arabia
}

Correspondence: Thomas J Webster Department of Chemical Engineering and Program in Bioengineering, Northeastern University, 360 Huntington Ave, Boston, MA, USA, 02II5

Email th.webster@neu.edu

\begin{abstract}
Excessive fibroblast adhesion and proliferation on the surface of medical implants (such as catheters, endotracheal tubes, intraocular lenses, etc) can lead to major postsurgical complications. This study showed that when coated on tissue culture polystyrene, lubricin, a nanostructured mucinous glycoprotein found in the synovial fluid of joints, decreased fibroblast density for up to 2 days of culture compared to controls treated with phosphate buffered saline (PBS). When examining why, similar antifibroblast density results were found when coating tissue culture polystyrene with bovine submaxillary mucin (BSM), an even smaller protein closely related to the central subregion of lubricin. Additionally, results from this study demonstrated that in contrast to BSM or controls (PBS-coated and non-coated samples), lubricin was better at preserving the health of nonadherent or loosely adherent fibroblasts; fibroblasts that did not adhere or loosely adhered on the lubricin-coated tissue culture polystyrene adhered and proliferated well for up to an additional day when they were reseeded on uncoated tissue culture polystyrene. In summary, this study provides evidence for the promise of nanostructured lubricin (and to a lesser extent BSM) to inhibit fibroblast adhesion and growth when coated on medical devices; lubricin should be further explored for numerous medical device applications.
\end{abstract}

Keywords: lubricin, antiadhesive, fibroblasts, mucin

\section{Introduction}

Fibroblast adhesion and growth can result in major postoperative complications. For example, one major complication following cataract surgery is posterior capsular opacification of implanted intraocular lenses due to the adhesion, migration, collagen deposition, and lens fiber generation from epithelial cells and fibroblasts that remain at the surgery site after the natural lens is removed. ${ }^{1}$ The impact is that additional procedures must be performed to remove the obstructions from the optical path of the lens. According to a study by Awasthi et $a{ }^{2},{ }^{2}$ the only effective procedure to treat this posterior capsular opacification is using a Nd:YAG (neodymium-doped yttrium aluminum garnet) laser capsulotomy to clear the visual axis. This procedure represents a significant cost burden to the national health care system and can result in retinal detachment, iris hemorrhage, corneal edema, extreme dislocation or damage of the implanted intraocular lenses; it can also exacerbate localized endophthalmitis (infection of the inner eye). ${ }^{2}$

In addition to excessive fibroblast adhesion, unwanted extracellular matrix deposition is a major cause of the failure of other medical devices, including indwelling catheters, endotracheal tubes, and gastrointestinal stents. ${ }^{1-4}$ The deposition of extracellular matrix proteins on the surface of an implant can also act as a nidus for 
bacterial colonization., ${ }^{5,6}$ Tissue adhesions can also result in a wide range of clinical issues for patients following abdominal surgery, from abdominal discomfort to complete intestinal blockage. ${ }^{1-4}$ These tissue adhesions require surgery for adhesiolysis and are a major cause of morbidity. One study estimated that the economic burden of hospitalization from lower abdominal-pelvic adhesiolysis in the United States, including hospital and provider costs, was well over US\$1.18 billion. $^{1}$

Thus, there is a need to control unwanted cellular (mostly fibroblast) adhesion and extracellular matrix formation on numerous medical devices in a safe yet effective way that will not disrupt the function of the medical device itself. One such protein that may accomplish this is a nanostructured mucinous glycoprotein called lubricin. Lubricin is an extended polymeric protein less than $200 \mathrm{~nm}$ in length and capped with globular $\mathrm{N}$ and $\mathrm{C}$ termini. The extensive O-linked glycosylations in a central mucin domain enable amphipathic-like chemical behavior that provides a natural lubricating barrier layer within the body (eg, joints). ${ }^{7,8}$ For example, a study by Rhee et al showed that lubricin was able to prevent the adhesion of synoviocytes on tissue culture polystyrene plates. ${ }^{8}$ However, to date no one has investigated the role that lubricin could play in reducing fibroblast density, which is a much more significant clinical problem than reducing synoviocyte density. ${ }^{1-4}$ Thus, the hypothesis of this study was that lubricin could be used as a simple medical device coating to prevent fibroblast adhesion and growth, without adversely affecting the overall health of the unattached cells (which could lead to significant toxicity). Further, in order to start investigating the origin of lubricin's anticell adhesive properties, this study also examined the antifibroblast adhesive properties of bovine submaxillary mucin (BSM), which is an even smaller nanostructured mucin subregion of lubricin (less than $100 \mathrm{~nm}$ from the $\mathrm{N}$ to $\mathrm{C}$ termini). ${ }^{7,8}$

\section{Materials and methods Protein preparation}

The lubricin used in these trials was extracted under sterile conditions from bovine synovial fluid obtained from Pel-Freez Biologicals (Rogers, Arkansas, AR, USA). ${ }^{7}$ The sterile extraction procedure was described fully by Jay et al, but in short, it involved the extraction of lubricin from bovine synovial fluid through a hyaluronate digestion, followed by anion exchange chromatography and affinity chromatography to purify and concentrate the protein. ${ }^{7}$ Lubricin and BSM (Sigma-Aldrich, St Louis, MO, USA) were used at a concentration of $200 \mu \mathrm{g} / \mathrm{mL}$. Prior to use, the BSM solutions were filtered through low protein binding sterile filters with a pore size of $0.2 \mu \mathrm{m}$ (Corning, NY, USA). Sterile phosphate buffered saline (PBS) was used in the dilution process for both lubricin and BSM.

\section{Qualitative fibroblast density}

Twenty-four well polystyrene plates (Thermo Fisher Scientific, Waltham, MA, USA) were coated with $125 \mu \mathrm{L}$ of lubricin, BSM, or PBS (controls) and the treated plates were rocked overnight at room temperature to ensure a uniform coating. Fibroblast cells were chosen for this study due to their ubiquitous nature in the body, relatively high growth rate, and strong adhesive properties. Human fibroblast cells (ATTC\# CCL-110) were grown in tissue culture flasks in Eagle's Minimal Essential Medium (EMEM) containing 10\% fetal bovine serum (FBS) and 1\% penicillin/streptomycin (all supplies from Life Technologies [Carlsbad, CA, USA]). Once fibroblasts reached confluence on the tissue culture flasks, trypsin (Sigma-Aldrich) was used to suspend the cells; the cells were then counted using a Beckman Coulter counter (Beckman Coulter Inc., Brea, CA, USA). The cells were diluted to $7 \times 10^{3}$ cells per $\mathrm{mL}$ and $1 \mathrm{~mL}$ of the solution was added to each of the treated wells. The plates were then incubated overnight in $5 \% \mathrm{CO}_{2}$ at $37^{\circ} \mathrm{C}$. Phase contrast microscope images were taken at $5 \times$ and $10 \times$ after 1 day. On day 2 , the plates were rinsed with PBS and fresh media was added prior to a second set of images being taken. To determine the toxicity of the lubricin and BSM solutions, nonadherent or loosely adherent fibroblasts were reseeded onto uncoated tissue culture polystyrene plates on day 2 . After an additional day of culture, phase contrast images of the attached cells were once again acquired.

\section{Quantitative fibroblast density}

To quantify fibroblast numbers, an MTS kit (Sigma-Aldrich) was also used in the present study. For this, 96-well tissue culture plates (Thermo Fisher Scientific) were treated with $50 \mu \mathrm{L}$ of PBS, $0.2 \mathrm{mg} / \mathrm{mL}$ BSM, or $0.2 \mathrm{mg} / \mathrm{mL}$ lubricin. Blank controls were also used. The coatings were allowed to dry overnight similar to the procedure described in the previous section. Each well was then seeded with 80,000 cells/well and fibroblasts were cultured as described above for 1 and 2 days under standard conditions. At the end of the prescribed time period, the wells were rinsed to remove all of the nonadherent or loosely attached cells, then $30 \mu \mathrm{L}$ of an MTS dye was added to each well and the plate was incubated under standard conditions for an additional 4 hours. Absorbance values were obtained through a spectrophotometer (Spectramax; Molecular Devices, 
Sunnyvale, CA, USA) at a wavelength of $490 \mathrm{~nm}$. Absorbance readings at different cell concentrations $\left(2.5 \times 10^{3}\right.$, $1 \times 10^{4}, 4 \times 10^{4}, 8 \times 10^{4}$, and $1.15 \times 10^{5}$ cells $/ \mathrm{mL}$ ) were used to make a standard curve.

\section{Statistics}

All experiments were run in triplicate and repeated a minimum of three different times. Differences between the means were determined using a Student's $t$-test.

\section{Results}

\section{Cell adhesion and proliferation}

After 1 day of incubation, fibroblasts on untreated and PBS-soaked tissue culture plates showed healthy cell adhesion and preconfluent spreading, while cells plated on BSM-coated samples remained either unattached or very loosely attached and clumped; fibroblasts on lubricincoated samples were completely unattached or very loosely attached (Figure 1).

On the second day, the media in the wells was aspirated and the wells were rinsed once with PBS, then fresh media was added. The untreated and PBS-coated wells demonstrated typical fibroblast attachment and proliferation (Figure 2A and B). However, similar to day 1, fibroblast clumps were found for the BSM-coated wells (Figure 2C). For the case of the lubricin-coated samples, similar to day 1 , fibroblasts were unattached or very loosely attached; thus, all cells were removed when changing media and none appear in the image (see Figure 2D).

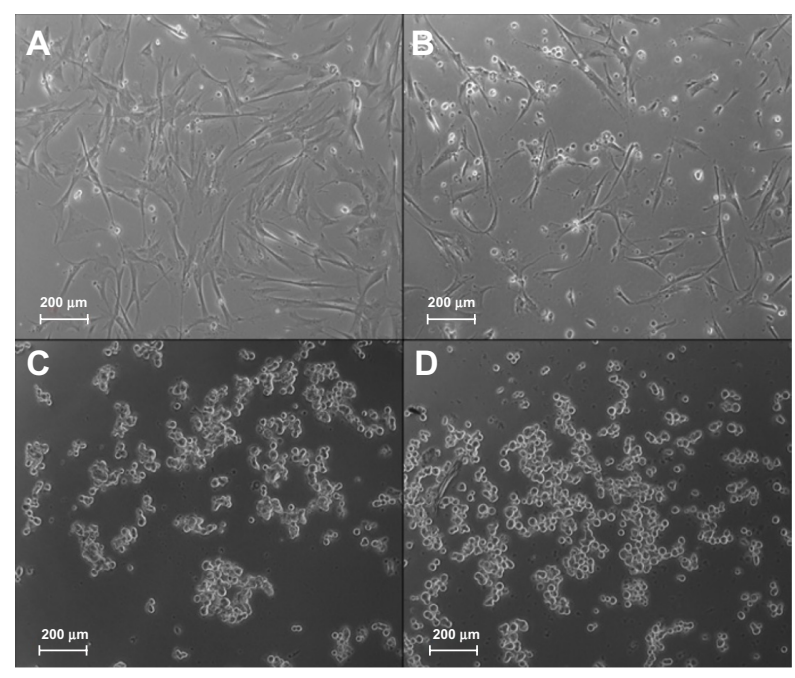

Figure I Day I phase contrast images of fibroblasts grown on tissue culture plates Notes: (A) Untreated; (B) PBS-coated; (C) BSM-coated; (D) lubricin-coated. Scale bars on bottom of each image.

Abbreviations: PBS, phosphate buffered saline; BSM, bovine submaxillary mucin.

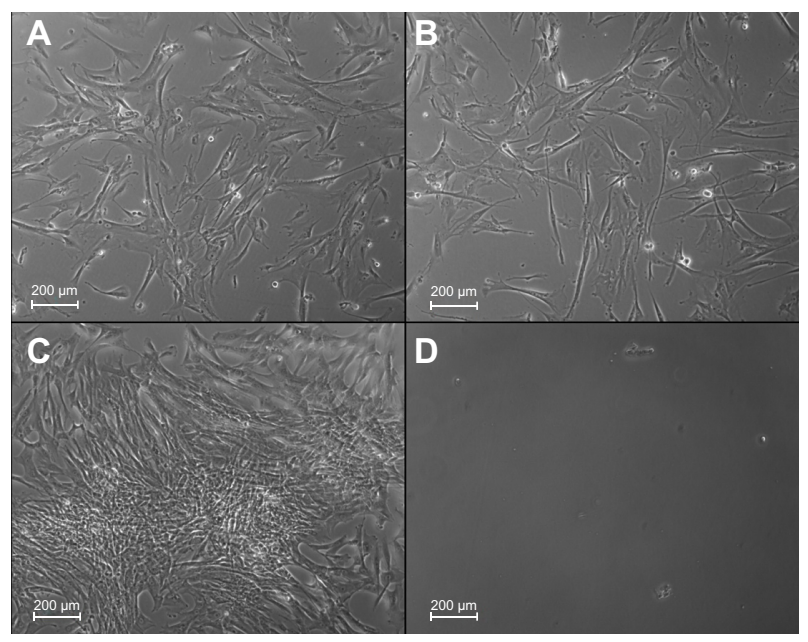

Figure 2 Day 2 phase contrast images of fibroblasts grown on tissue culture plates. Notes: (A) Untreated; (B) PBS-coated; (C) BSM-coated; (D) lubricin-coated. Scale bars on bottom of each image.

Abbreviations: PBS, phosphate buffered saline; BSM, bovine submaxillary mucin.

As mentioned, the aspirates before the second-day trials were reseeded on fresh sterile uncoated plates after being exposed to lubricin or BSM. The fibroblasts from the uncoated and PBS-coated wells behaved in a similar manner; there was very little adhesion and the wells were filled mostly with floating cells (Figure 3A and B). Similarly, the nonadherent or very loosely adherent fibroblasts from the BSM-coated wells showed some clumping and adhesion, but were mostly nonadherent after reseeding (Figure 3C). In contrast, the wells containing fibroblast aspirates from the lubricin-coated plates showed a great deal of adhesion and cell spreading (Figure 3).

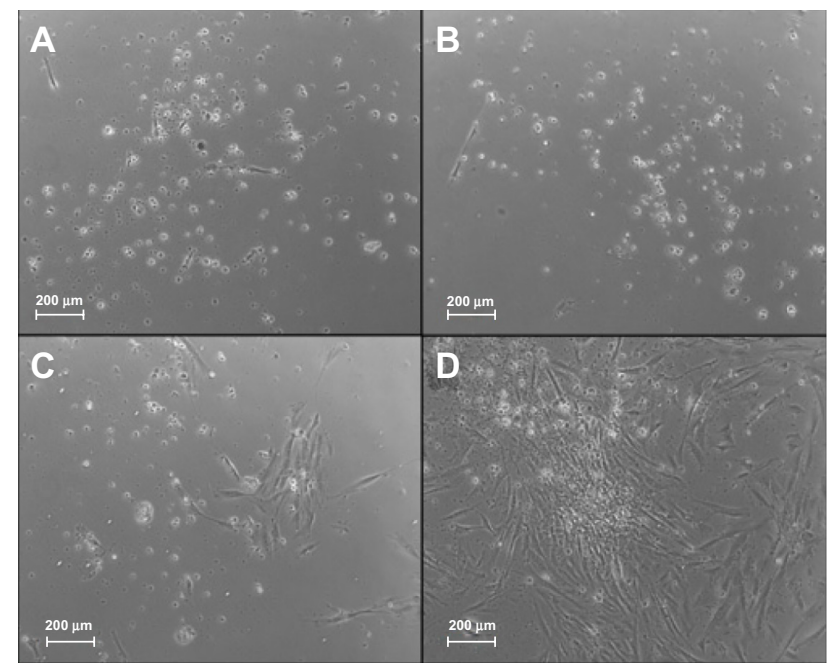

Figure 3 Day I phase contrast images of fibroblast aspirated and reseeded from 2 days of culture.

Notes: (A) Untreated; (B) PBS-coated; (C) BSM-coated; (D) lubricin-coated tissue culture polystyrene. Cells after reseeding, cells were cultured for an additional day before images were taken.

Abbreviations: PBS, phosphate buffered saline; BSM, bovine submaxillary mucin. 


\section{MTS assays}

The MTS trials confirmed the qualitative results and showed that both lubricin and BSM significantly decreased fibroblast densities when compared to the PBS-coated samples (Figure 4). In fact, compared to PBS-coated controls, results demonstrated that lubricin and BSM-coated tissue culture polystyrene decreased fibroblast density by approximately $10 \%$ and $30 \%$ after just 1 day and approximately $30 \%$ after just 2 days of culture, respectively. BSM inhibited fibroblast density better than lubricin after 1 day; however, both proteins equally inhibited fibroblast density after 2 days of culture. Interestingly, adding BSM and lubricin to the cell culture media completely inhibited fibroblast growth on the tissue culture polystyrene after 1 day and both inhibited fibroblast density approximately $30 \%$ compared to PBS controls after 2 days of culture.

\section{Discussion}

The results of these trials showed that lubricin (and to a lesser extent mucin) acted as an effective nontoxic surface treatment to reduce fibroblast density. Lubricin-coated surfaces completely blocked full fibroblast adhesion and proliferation however, whenever reseeded, the nonadherent fibroblasts were still able to grow when reseeded in a healthy normal manner. Unattached or loosely attached fibroblasts were unable to grow while in the presence of these adherent biomolecules. While the BSM prevented the initial firm adhesion of fibroblasts after the first day of culture, cells formed clumps on the second day and began to stick and proliferate on the surface. Moreover, after reseeding, fibroblasts from the BSM-coated tissue culture polystyrene were not able to attach firmly and grow as quickly as cells that were nonadherent or loosely adherent on the lubricin-coated plates.

There are a number of future directions for the present study. Adhesion and prolonged proliferation of fibroblasts can lead to excessive and detrimental fibrous encapsulation on implants designed for bladder, vascular, and orthopedic applications. ${ }^{9}$ Stopping the initial fibroblast adhesion in a nontoxic manner could lead to improved implant health and better patient outcomes. Additionally, studies have shown that natural lubricin production may aid in the prevention of unwanted cellular adhesion and tissue constrictions around medical implants..$^{10}$ The work of Cheriyan et al indicated lubricin protein production around implanted breast tissue expanders may both prevent unwanted tissue adhesion and result in the creation of a boundary layer which can protect the surrounding tissue by dissipating the frictional and shear forces at the surface of the implant. ${ }^{10}$ This indicates that lubricin is not only a biocompatible surface coating but that it may also be part of the natural process of reducing undesirable surface interactions between an implant and surrounding tissue.

It is also intriguing to ponder what role the nanostructure orientation of lubricin is playing in these responses. In an initial attempt to determine this role, the present study examined BSM as a mimic of the $100 \mathrm{~nm}$ mucin-like subregion of lubricin; however, we did not find exactly the same results

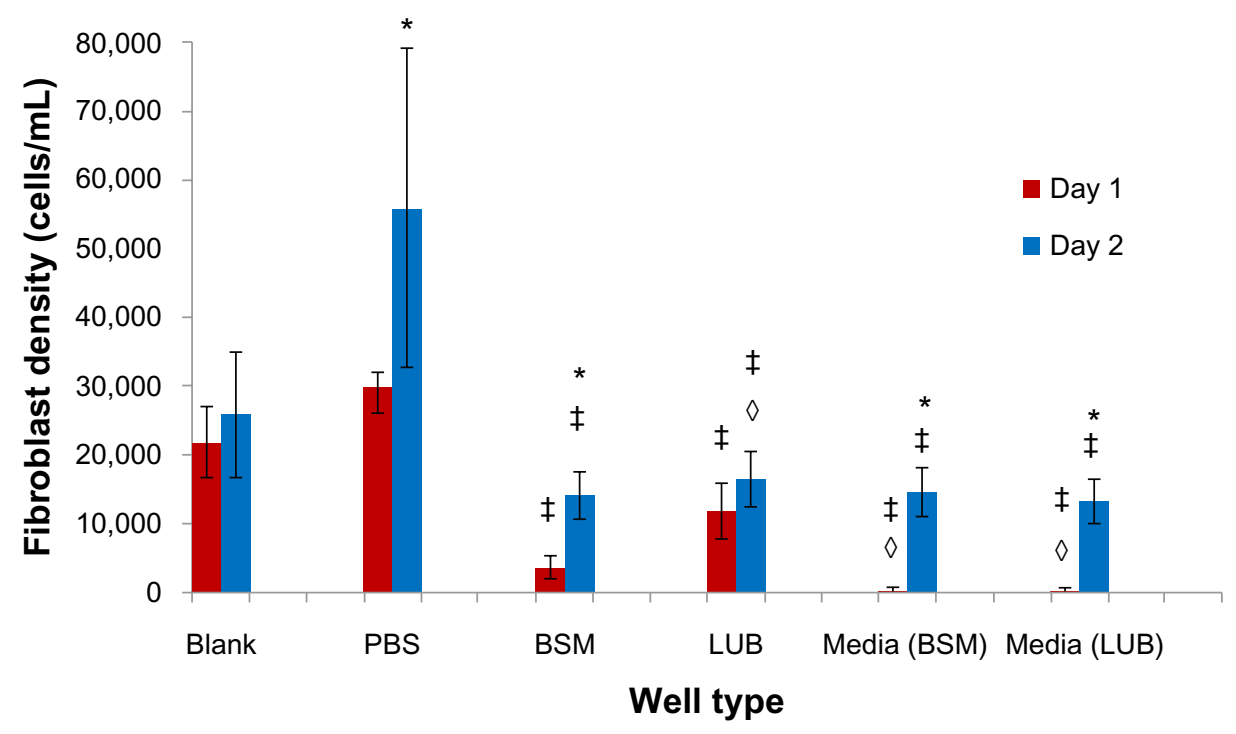

Figure 4 Decreased fibroblast density on BSM- and lubricin-coated tissue culture polystyrene as determined using the MTS assay.

Notes: Original seeding density was 80,000 cells per sample. ${ }^{*} P<0.05$ comparing day 2 to day I on the same sample. Both lubricin- and BSM-coated wells experienced significantly lower $(P<0.05)$ cell density compared to PBS after I and 2 days of culture; ${ }^{\ddagger}<0.01$ compared to PBS at the same day; ${ }^{\circ} P<0.0 \mathrm{I}$ compared to BSM at the same day. Media (BSM and lubricin) refers to media containing these respective proteins.

Abbreviations: PBS, phosphate buffered saline; BSM, bovine submaxillary mucin; LUB, lubricin; MTS, 3-(4,5-dimethylthiazol-2-yl)-5-(3-carboxymethoxyphenyl)-2-(4sulfophenyl)-2H-tetrazolium. 
in terms of fibroblast inhibition and maintenance of viability after exposure. This report indicates that mucin may be part of the answer but the mucin domain of lubricin is not the only reason for these effects. At the nanoscale, the role of lubricin conformation also deserves some attention. For example, while the tissue culture polystyrene used in the present study was smooth at the nanoscale, studies have determined that nano-rough polymer surfaces minimize fibroblast density without the use of nanoscale protein coatings. ${ }^{9}$ It was speculated in that study that nanoscale surface features alter surface energetics to promote the adsorption of hydrophilic proteins that are known to decrease fibroblast functions. ${ }^{9}$ It is possible that a combined effect of nanoscale roughness with a lubricin coating could even further decrease fibroblast functions, which should also be the focus of future studies, especially since numerous studies have shown the ability of nanostructured surface features to promote protein bioactivity. ${ }^{10-13}$ Additionally, it is possible to express human lubricin in Chinese hamster ovary cells as a recombinant protein. Future studies should also use recombinant human lubricin as a more readily available source of lubricin. ${ }^{14}$

\section{Conclusion}

This study showed that when coated on tissue culture polystyrene, lubricin, a nanostructured mucinous glycoprotein found in the synovial fluid of joints, decreased fibroblast density for up to 2 days of culture. Similar antifibroblast density results were found when coating tissue culture polystyrene with BSM, an even smaller protein closely related to the central subregion of lubricin. In addition, results from this study demonstrated that in contrast to BSM or controls (PBS and non-coated samples), lubricin preserved the viability of nonadherent or loosely adherent fibroblasts - fibroblasts that did not adhere or loosely adhered on the lubricin-coated tissue culture polystyrene became adherent and proliferated well for up to one additional day of culture when they were reseeded on uncoated tissue culture polystyrene. In summary, this study provided significant evidence for the promise of lubricin to inhibit fibroblast adhesion and growth in a nontoxic manner when coated on synthetic surfaces. Lubricin may therefore be a viable cellular antiadhesive option for numerous medical device surfaces.

\section{Acknowledgments}

The authors would like to thank Stanley Chung for aid in collecting microscope images and Northeastern University for funding this research.

\section{Disclosure}

Gregory D Jay holds patents that relate to lubricin. The other authors report no conflicts of interest in this work.

\section{References}

1. DeCherney AH, diZerega GS. Clinical problem of intraperitoneal postsurgical adhesion formation following general surgery and the use of adhesion prevention barriers. Surg Clin North Am. 1997;77(3): 671-688.

2. Awasthi N, Guo S, Wagner BJ. Posterior capsular opacification: A problem reduced but not yet eradicated. Arch Ophthalmol. 2009; 127(4):555-562.

3. Cordeiro MF, Chang L, Lim KS, et al. Modulating conjunctival wound healing. Eye (Lond). 2000;14(Pt 3B):536-547.

4. Binda MM, Molinas CR, Koninckx PR. Reactive oxygen species and adhesion formation: Clinical implications in adhesion prevention. Hum Reprod. 2003;18(12):2503-2507.

5. Pavithra D, Doble M. Biofilm formation, bacterial adhesion and host response on polymeric implants - issues and prevention. Biomed Mater. 2008;3(3):034003.

6. Hauck CR, Agerer F, Muenzner P, Schmitter T. Cellular adhesion molecules as targets for bacterial infection. Eur J Cell Biol. 2006;85(3-4): 235-242.

7. Jay GD, Haberstroh K, Cha CJ. Comparison of the boundary-lubricating ability of bovine synovial fluid, lubricin, and Healon. J Biomed Mater Res. 1998;40(3):414-418.

8. Rhee DK, Marcelino J, Baker M, et al. The secreted glycoprotein lubricin protects cartilage surfaces and inhibits synovial cell overgrowth. J Clin Invest. 2005;115(3):622-631.

9. Vance RJ, Miller DC, Thapa A, Haberstroh KM, Webster TJ. Decreased fibroblast cell density on chemically degraded poly-lactic-co-glycolic acid, polyurethane, and polycaprolactone. Biomaterials. 2004;25(11): 2095-2103.

10. Cheriyan T, Guo L, Orgill DP, Padera RF, Schmid TM, Spector M. Lubricin in human breast tissue expander capsules. J Biomed Mater Res B Appl Biomater. 2012;100(7):1961-1969.

11. Webster TJ, Schadler LS, Siegel RW, Bizios R. Mechanisms of enhanced osteoblast adhesion on nanophase alumina involve vitronectin. Tissue Eng. 2001;7(3):291-301.

12. Taylor E, Webster TJ. Reducing infections through nanotechnology and nanoparticles. Int J Nanomedicine. 2011;6:1463-1473.

13. Yang L, Zhang L, Webster TJ. Nanobiomaterials: state of the art and future trends. Advanced Engineering Materials. 2011;13(6): B197-B217.

14. Flannery CR, Zollner R, Corcoran C, et al. Prevention of cartilage degeneration in a rat model of osteoarthritis by intraarticular treatment with recombinant lubricin. Arthritis Rheum. 2009;60(3):840-847.
International Journal of Nanomedicine

\section{Publish your work in this journal}

The International Journal of Nanomedicine is an international, peerreviewed journal focusing on the application of nanotechnology in diagnostics, therapeutics, and drug delivery systems throughout the biomedical field. This journal is indexed on PubMed Central, MedLine, CAS, SciSearch $\AA$, Current Contents ${ }^{\circledR} /$ Clinical Medicine,

\section{Dovepress}

Journal Citation Reports/Science Edition, EMBase, Scopus and the Elsevier Bibliographic databases. The manuscript management system is completely online and includes a very quick and fair peer-review system, which is all easy to use. Visit http://www.dovepress.com/ testimonials.php to read real quotes from published authors. 